\title{
In-ear microphone measures in the ear canal with bone conduction stimulation: An application for estimating a cross-talk compensation filter
}

\author{
Irwansyah* and Tsuyoshi Usagawa $^{\dagger}$ \\ Graduate School of Science and Technology, Kumamoto University, \\ 2-39-1 Kurokami, Chuo-ku, Kumamoto, 860-8555 Japan
}

(Received 30 September 2018, Accepted for publication 15 April 2019)

Keywords: In-ear microphone, Ear-canal sound pressure, Bone conduction, Cross-talk compensation filter PACS number: 43.38.-p, 43.38.Kb, 43.50.Ki, 43.64.Jb [doi:10.1250/ast.41.439]

\section{Introduction}

Bone-conduction (BC) transducer has been known for years as a tool to help patients with problems in their outer or middle ears. It works by transmitting sound by bone vibration directly to the cochlea in the inner ear. While BC technology is usually used in hearing aids, its applications are not limited to this alone. Recently, interest in "skin-drive" BC transducers has increased [1] and are being applied in some areas such as for music [2] or military communication [3]. Since implantation is not required, they are more acceptable and can be used for people with normal hearing.

Unlike air-conduction (AC) sound, presenting bone-conduction (BC) sound from a single $\mathrm{BC}$ transducer on the head will result in the sound reaching the cochlea in both ears. As a result, small level differences in hearing thresholds between the cochleae were observed [4]. Since BC stimulation at a single location on the head can reach both cochleae, this "cross-talk" phenomenon is considered by researchers as one of the factors that may reduce the ability of a listener to sense sound direction when using a bilateral BC device [5-7].

Several studies have been carried out concerning how to eliminate or reduce the cross-talk and its effect [8-11]. One of the suitable ways is by implementing a cross-talk cancellation system [9]. By measuring transfer functions on routes from BC transducers to the cochleae in the dry human skull, an experimental study reported that the cross-talk cancellation system worked well in the skull at frequencies from $250 \mathrm{~Hz}$ to $1.5 \mathrm{kHz}$ with the cross-talk signal attenuation of up to $12 \mathrm{~dB}$ [9]. However, estimating BC transfer functions to apply the system to a human being properly is still an issue.

A method to estimate $\mathrm{BC}$ transfer functions has been proposed by Zhong in [10] and Mcleod in [11]. The phase and level of a pure-tone sound from an earphone are adjusted to cancel a sound from a BC transducer (i.e., resulting in perceived silence). This method, however, requires adjustment for each frequency component and therefore is too timeconsuming [11].

The closest position to the cochlea which may help to estimate BC signals at the cochlea with a minimally invasive technique is in the ear canal. When a stimulus is presented via

\footnotetext{
*e-mail: irwansyah@ieee.org

†e-mail: tuie@cs.kumamoto-u.ac.jp
}

a BC transducer, the ear-canal sound pressure level (ECSP) changes because of two causes. These are: first, vibrations of the surrounding structure of the ear canal [12] and second, otoacoustic emissions (OAEs) [13-15]. Reinfeldt et al. reported that changes in ECSP were relatively similar to the vibration of the cochlea [12]. Thus, ECSP data might allow the implementation of a cross-talk cancellation system.

In our previous work [16], it was shown that placing a miniature microphone in the ear canal and covering it by earmuffs, BC transfer functions could be estimated. A clinical microphone such as ER-7C probe microphone is normally used for measurement in the ear canal, but it is very costly [17]. Because BC transfer functions can easily change depending on the position of the $\mathrm{BC}$ transducer on the head [18] (implanted one might be more stable), from user's point of view, it would be desirable to have a compact, simple, inear microphone for easy use. In this paper, we assembled an in-ear microphone used for estimating BC transfer functions which are needed to estimate a cross-talk compensation filter. Cancellation tests with repeated measurements on the real ears were conducted to evaluate the performance of the proposed microphone for estimating a cross-talk compensation (CTC) filter.

\section{In-ear microphone assembly}

In [19,20], a combination of an earplug (or earphone shell) and a probe microphone is called as in-ear microphone. The in-ear microphone assembly consists of silicone tubes, plastic tube, soft silicone, triple flange silicone tip, earphone shell and subminiature microphone. The microphone used in this study was a subminiature omnidirectional condenser lavalier microphone AT-899. To measure the ECSP, the microphone was fitted with three silicone tubes which have different diameters and lengths (see Step 1 of Fig. 1). The microphone was then put in a plastic tube (see Step 2 of Fig. 1). To isolate the microphone from any undesired AC sounds, the plastic tube was filled with soft silicone (see Step 3 of Fig. 1). A 2-mm diameter probe tube is used as a transmission path between the microphone and an earphone shell placed in the ear canal (see Step 4 of Fig. 1). To minimize the occlusion effect [21], a small-size triple flange silicone tip was used to enable a deeper insertion of ear tip (about $12 \mathrm{~mm}$ ), and an additional probe tube was also used to allow sounds to escape (see Step 4 of Fig. 1). The main purpose of using the silicone tip is to 


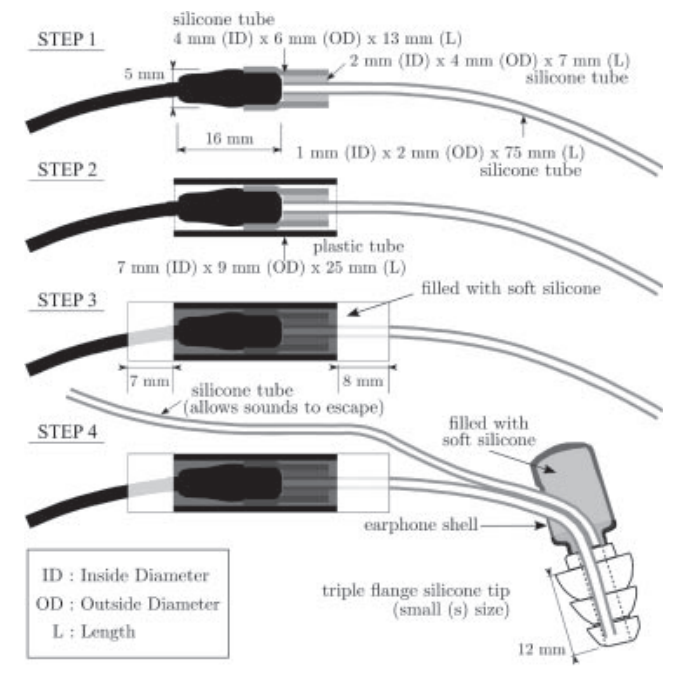

Fig. 1 Details of the in-ear microphone assembly used for ECSP measurements.

reduce air-borne radiation from $\mathrm{BC}$ transducers [22]. According to the specification, the frequency response of the microphone should be flat [23]. Since the modification is required, there is a possibility that the response is not flat anymore. However, it is still acceptable because our measurements are about level difference or attenuation, so that correction is no longer required.

\section{Instrumentation}

The bone conduction headphone, Goldendance MGD-01, was used to produce sound stimuli. The headphone amplifier, Neoteck NTK059, was employed due to low impedance of BC actuator. For recording, the microphone Audio Technica AT-899 equipped with a power module Audio Technica AT8539 and also using a pre-amplifier TASCAM US-20x20 with a phantom power source to obtain a better sensitivity of the microphone. TASCAM US-20x20 has a USB3.0 computer connection, so that recorded signals were stored in a laptop with Linux operating system. Figure 2 shows the scheme of the measurement system setup.

\section{Measurements}

We conducted two types of experiments in order to evaluate the performance of the in-ear microphone.

\subsection{Participants}

Three participants (males) with self-reported normal hearing participated in the experiments. The age range is between 26 and 35 years old.

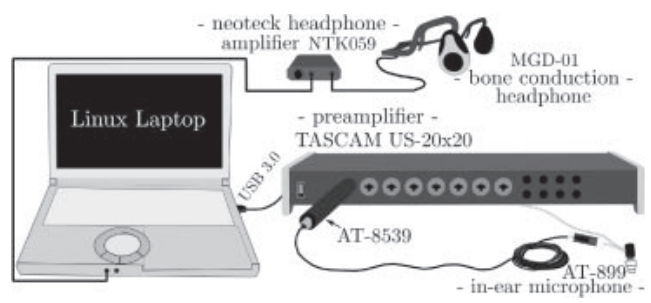

Fig. 2 Scheme of the measurement system setup.

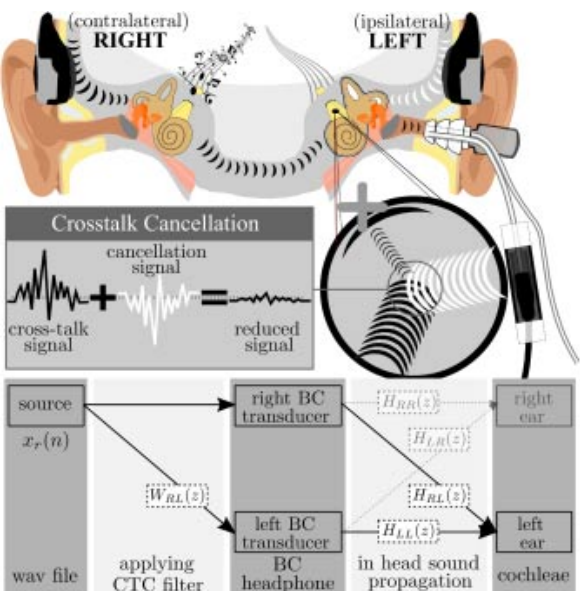

Fig. 3 Schematic diagram and its illustration of the oneside cross-talk cancellation system used to minimize a cross-talk signal in the left ear.

\subsection{Procedure}

Experiments were performed in an anechoic chamber with the background noise level of $20 \mathrm{~dB}$. The Rion NL-32 product was used as a sound level meter. Participants wore the MGD$01 \mathrm{BC}$ headphone. BC transducers of the headphone were placed slightly above the temporomandibular joint. The BC transducer was driven at a level corresponding to $60 \mathrm{~dB}$ SPL adjusted based on a loudness balancing technique [15]. The in-ear microphone was placed $12 \mathrm{~mm}$ (until the third layer of the ear tip) in the ear canal. The sampling rate used was $16 \mathrm{kHz}$. An illustration of the setup is shown in Fig. 3.

4.3. Experiment 1: BC transfer function

Since directly measuring $\mathrm{BC}$ signal at the cochlea is not possible, in this paper, the $\mathrm{BC}$ transfer function is defined as a transfer function from the BC transducer's input to ECSP's output. Although nonlinearity between eardrum vibration and response of cochlea is well known, we assumed that some extent of linearity holds for a certain range of BC actuator's input. The time-stretched pulse (TSP) sound was used as a stimulus to estimate $\mathrm{BC}$ transfer functions which are $H_{\mathrm{LL}}(z)$, ipsilateral, and $H_{\mathrm{RL}}(z)$, contralateral (see Fig. 3). This measurement was repeated ten times in which the in-ear microphone was being reinserted into the ear canal between repeated measures for each participant.

\subsection{Experiment 2: Cross-talk cancellation}

Experiment 2 used the same procedure as Experiment 1, but with an extra step. $H_{\mathrm{LL}}(z)$ and $H_{\mathrm{RL}}(z)$ were used to estimate a cross-talk compensation (CTC) filter, $W_{\mathrm{RL}}(z)$, using the filtered-x least mean square (FxLMS) algorithm. An adaptive CTC filter is required to handle the time-variant characteristics between $\mathrm{BC}$ transducers and the in-ear microphone. Among various adaptive algorithms, the FxLMS is the most suitable because of its computational simplicity and capability to include a secondary path $H_{\mathrm{LL}}(z)$ for estimating optimum filter coefficients. Pink noise as a test stimulus was then filtered by the CTC filter to estimate the cancellation signal. After that, we presented the stimulus with and without cancellation via the $\mathrm{BC}$ headphone to be recorded by the inear microphone in the left ear canal. As suggested in [15], the 


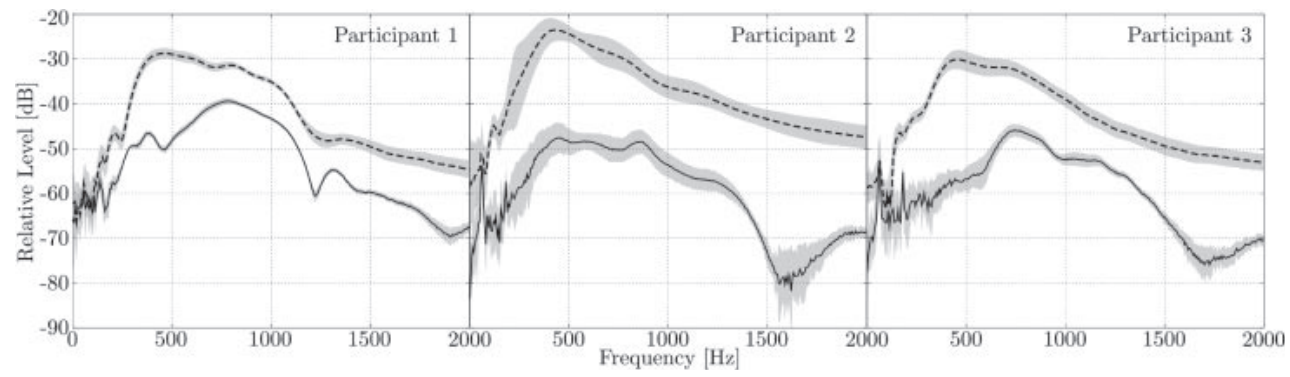

Fig. 4 Frequency responses of ipsilateral $\mathrm{BC}$ transfer function $H_{\mathrm{LL}}(z)$ and contralateral $\mathrm{BC}$ transfer function $H_{\mathrm{RL}}(z)$. Means are shown as dashed line (- - ) for $H_{\mathrm{LL}}(z)$ and solid line $(-)$ for $H_{\mathrm{RL}}(z)$ while standard deviations are provided as band around the lines.
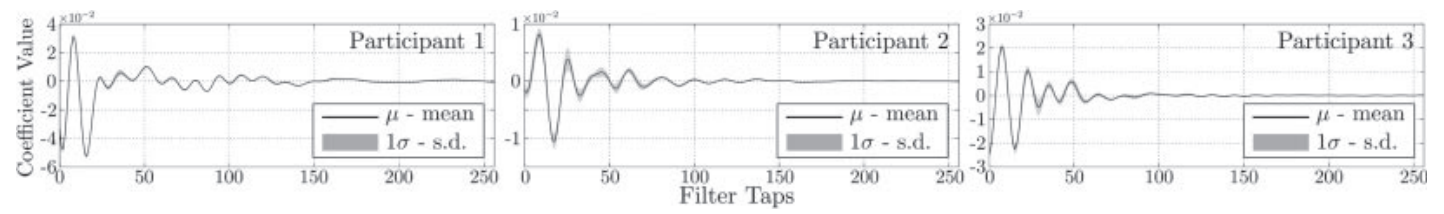

Fig. 5 Impulse response of estimated $10 \mathrm{CTC}$ filters shown as the mean $(\mu)$ with standard deviation $(\sigma)$ for each participant.

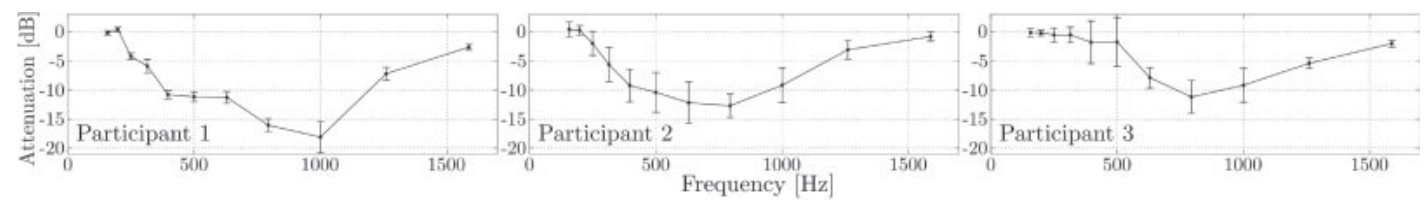

Fig. 6 Attenuation of the cross-talk sound in the left ear of each participant.

stimulus was at a level corresponding to $60 \mathrm{~dB}$ sound pressure level (SPL) which is not harmful to hearing. The recorded sounds were analyzed in each one-third-octave band between $160 \mathrm{~Hz}$ and $1.6 \mathrm{kHz}$ to obtain the attenuation level. The whole process was repeated ten times for each participant.

\section{Results and discussions}

\subsection{Experiment $1-\mathrm{BC}$ transfer functions}

As discussed in [11], low inter-cochlear level differences occurred mostly at low frequencies. Figure 4 shows frequency responses of $\mathrm{BC}$ transfer functions observed at low frequencies for ten times measurements using the in-ear microphone placed in the left ear canal of each participant. From Fig. 4, it can be seen that the level difference between $H_{\mathrm{LL}}(z)$ and $H_{\mathrm{RL}}(z)$ in $\mathrm{dB}$ is about $5-25 \mathrm{~dB}$ depending on the frequency, which is quite high (ipsilateral side is always larger than contralateral in the observed frequencies). One of the reasons might be the position of the $\mathrm{BC}$ transducer that was placed above temporomandibular joint where the skin is quite thick compared to that at the mastoid. Thus, the vibration propagating through the skin might be quite dominant and was recorded by the microphone, but the cross-talk sound from the contralateral $\mathrm{BC}$ transducer was still able to be observed and could be felt by the participants. Repeated measurements with the position of the $\mathrm{BC}$ transducers remaining the same, showed that the frequency responses were quite stable with the standard deviation of up to $12 \mathrm{~dB}$ for frequencies below $2 \mathrm{kHz}$ when the in-ear microphone is reinserted several times. In addition, due to physiological noise at around $125 \mathrm{~Hz}$ [24], a low-cut filter was applied in all measurements.

\subsection{Experiment 2 - Cross-talk cancellation}

Using the estimated BC transfer functions obtained by Experiment 1, the cross-talk compensation filters were able to be estimated for all participants. Figure 5 shows the estimated filter of each participant in which the standard deviation of coefficient values was quite small. It means that the microphone was quite stable against a small change in position when the earphone shell is reinserted ten times. Even though the estimated filter was quite stable, there was a large variation in the standard deviations of the attenuation level of the cross-talk sound in the left ear as shown in Fig. 6. This variation might be due to a human body movement or jaw position which is considered in [11] as a potential problem for the development of a cross-talk cancellation system. A related study on human cadaver heads found that at frequencies between 0.5 and $1 \mathrm{kHz}$ the head acts as a mass-spring system [25]. On the contrary, it might be regarded as a nonlinear system at higher frequencies above $1.5 \mathrm{kHz}$ according to the study on the dry human skull [9]. We therefore suspect that the better attenuation performance at the mid-range frequency as shown in Fig. 6 is observed because that frequency range is best suited for linear adaptive filtering algorithms such as 
FxLMS. In addition, physiological noise such as from breathing (respiration) or blood flow can cause the ear canal wall vibrates especially when the ear canal is occluded [26]. It occurs only at low frequencies around $125 \mathrm{~Hz}$ in [24] or even higher up to $250 \mathrm{~Hz}$ as reported in [26]. Therefore, we activated the analog low-cut filter offered by the microphone power module AT-8539 to minimize the effect of the noise on the estimation of the CTC filter. As a result, the cancellation did not work well at frequencies below around $200 \mathrm{~Hz}$ in our experiments. However, further investigation is still necessary. In general, experiments on the cross-talk cancellation showed that the sound reduction was observed by the microphone. Participants also reported that they could feel a decrease in the perceived loudness as well as a change in the perceived direction.

\section{Conclusions}

This paper discusses use of an in-ear microphone for estimating a cross-talk compensation (CTC) filter by which a "cross-talk" sound from the contralateral bone conduction (BC) transducer is canceled by an "anti-sound" from the ipsilateral one. An earphone shell with a triple flange silicone tip is inserted into the ear canal and connected to the microphone placed outside by a $2-\mathrm{mm}$ diameter probe tube. To estimate the CTC filter, a change of ear-canal sound pressure (ECSP) is assumed to be relative to the vibration of the cochlea, so that $\mathrm{BC}$ transfer functions are possible to be estimated. By using the transfer functions, the CTC filter can be obtained as a result of the filtered-x least mean square (FxLMS) algorithm. Experimental results show that the microphone was stable to estimate the CTC filter while being reinserted between repeated measures. Sound reduction was observed by the microphone when a pink noise with its antisound was presented through BC transducers. The three participants also reported that they could feel a decrease in the perceived loudness.

As a future work, we also need to evaluate whether antisound used to reduce a cross-talk sound in the ipsilateral ear would have a positive or negative impact on the sound in the contralateral ear. A change of direction in the perceived sound is necessary to be investigated too, to check whether or not a cross-talk cancellation system will improve binaural hearing perception.

\section{References}

[1] S. Reinfeldt, B. Håkansson, H. Taghavi and M. Eeg-Olofsson, "New developments in bone-conduction hearing implants: A review," Med. Devices (Auckl.), 8, 79-93 (2015).

[2] https://aftershokz.com/ (Accessed 10 Apr. 2019).

[3] M. Blue, M. McBride, R. Weatherless and T. Letowski, "Impact of a bone conduction communication channel on multichannel communication system effectiveness," Hum. Factors, 55, 346-355 (2013).

[4] S. Stenfelt, "Transcranial attenuation of bone-conducted sound when stimulation is at the mastoid and at the bone conduction hearing aid position," Otol. Neurol., 33, 105-114 (2012).

[5] A. F. M. Snik, A. J. Beynon, E. A. M. Mylanus, C. T. M. van der Pouw and C. W. R. J. Cremers, "Binaural application of the bone-anchored hearing aid," Ann. Otol. Rhinol. Laryngol., 107, 187-193 (1998).

[6] J. Mejia, C. V. Nguyen, I. Yeend, T. Loi, R. Cowan and H.
Dillon, "Bone-conduction hearing aids: Into the (near) future," Hear. J., 68, 30-32 (2015).

[7] B. N. Walker and R. M. Stanley, "Evaluation of boneconduction headsets for use in multitalker communication environments," Proc. Hum. Factors Ergon. Soc. Annu. Meet., 49, 1615-1619 (2005).

[8] M. Iwaki and Y. Chigira, "Compensation of sound source direction perceived through consumer-grade bone-conduction headphones by modifying ILD and ITD," IEEE Int. Conf. GCCE, pp. 1-4 (2016).

[9] C. Liao, "Application of cross-talk cancellation to the improvement of binaural directional properties for individuals using bone anchored hearing aids (BAHA)," Thesis, Chalmers University of Technology (2010).

[10] X. Zhong and J. Dong, "Method and system for implementing stereo audio using bone conduction transducers," US Patent, 8989417 (2015).

[11] R. W. J. Mcleod and J. F. Culling, "Measurements of intercochlear level and phase differences of bone-conducted sound," J. Acoust. Soc. Am., 141, 3421-3429 (2017).

[12] S. Reinfeldt, S. Stenfelt and B. Håkansson, "Estimation of bone conduction skull transmission by hearing thresholds and ear-canal sound pressure," Hear. Res., 299, 19-28 (2013).

[13] D. W. Purcell, H. Kunov and W. Cleghorn, "Estimating bone conduction transfer functions using otoacoustic emissions," J. Acoust. Soc. Am., 114, 907-918 (2003).

[14] F. Kandzia, J. Oswald and B. Janssen, "Binaural measurement of bone conduction click evoked otoacoustic emissions in adults and infants," J. Acoust. Soc. Am., 129, 1464-1474 (2011).

[15] X. Qin and T. Usagawa, "Frequency characteristics of bone conduction actuators: Measurements of loudness and acceleration," Appl. Acoust., 126, 19-25 (2017).

[16] Irwansyah and T. Usagawa, "Application of active control technique on a bone conduction headphone for estimating a cross-talk compensation filter," Proc. IEEE Region 10 Conf., pp. 3099-3104 (2017).

[17] https://www.etymotic.com/auditory-research/microphones/er7c.html (Accessed 10 Apr. 2019).

[18] S. Stenfelt, "Simultaneous cancellation of air and bone conduction tones at two frequencies: Extension of the famous experiment by von Békésy,” Hear. Res., 225, 105-116 (2007).

[19] N. Westerlund, M. Dahl and I. Claesson, "In-ear microphone techniques for severe noise situations," Blekinge Inst. Technol., Research Report, 12 (2005).

[20] G. Bulbuller, M. P. Fargues and R. Vaidyanathan, "In-ear microphone speech data segmentation and recognition using neural networks," Proc. IEEE Workshop Digit. Signal Process., pp. 262-267 (2006).

[21] S. Stenfelt and S. Reinfeldt, "A model of the occlusion effect with bone-conducted stimulation," Int. J. Audiol., 46, 595-608 (2007).

[22] G. R. Lightfoot, "Air-borne radiation from bone conduction transducers,” Br. J. Audiol., 13, 53-56 (1979).

[23] www.audio-technica.co.jp/items/contents/102/model/AT899/ donwload/AT899.pdf (Accessed 10 Apr. 2019).

[24] E. H. Berger, "Preferred methods for measuring hearing protector attenuation," Proc. Inter-Noise, (2005).

[25] S. Stenfelt and R. L. Goode, "Transmission properties of bone conducted sound: Measurements in cadaver heads," J. Acoust. Soc. Am., 118, 2373-2391 (2005).

[26] E. H. Berger and J. E. Kerivan, "Influence of physiological noise and the occlusion effect on the measurement of real-ear attenuation at threshold," J. Acoust. Soc. Am., 74, 81-94 (1983). 